\title{
Exploitation des eaux minérales carbo-gazeuses
}

\author{
J.L. Honegger et A. Gadalia \\ ANTEA, groupe BRGM, Direction de l'eau
}

\section{D PROBLÉMATIQUE GÉNÉRALE}

\subsection{Introduction}

Un forage recaptant un aquifère peut être assimilé à un réacteur bio-physico-chimique. En effet il a pour fonction primaire de permettre le transport de fluide d'un niveau à un autre, mais ceci induit des changements importants dans les conditions thermodynamiques contrôlant ce fluide.

D'un point de vue physique il ne s'agit généralement que d'une évolution de pression qui, pour les profondeurs habituelles d'un forage d'eau plate, n'a pas de répercussions conséquentes sur les équilibres chimiques au cours de la remontée de l'eau vers la surface. Cette dépressurisation induit par contre des répercussions importantes dès que ces forages atteignent des profondeurs supérieures à quelques centaines de mètres ou bien que les eaux présentent des pressions partielles de gaz permettant l'expression de ceux-ci.

Cependant de nombreux autres paramètres sont à prendre en compte sur ce trajet; on peut citer parmi eux :

- la superposition et le captage presque systématique d'horizons producteurs différents, ayant chacun leur propre chimie,

- les perturbations hydrodynamiques (filtration,...) des eaux passant au travers des massifs de gravier, des crépines de forage et des crépines de pompe,

- la repressurisation par pompe immergée, avec les variations de vitesse et de pression qui en résultent,
- les interactions fluide/matériau (corrosion, sorption,...) au cours de la remontée.

En ce qui concerne la biologie des eaux, le captage d'une eau souterraine amène un changement de biotope, avec toutes ses conséquences bactériologiques.

Nous évoquerons ici les difficultés liées à la mise en production des eaux naturellement carbo-gazeuses.

\subsection{Généralités}

Une eau est considérée comme carbogazeuse si elle contient plus de $250 \mathrm{mg} / \mathrm{l}$ de $\mathrm{CO}_{2}$ libre (RAMBAUD, 1992). Cette valeur est surtout indicative car elle dépend des conditions thermodynamiques (température, pression mais aussi composition, $\mathrm{pH}$, potentiel d'oxydo-réduction,...) du fluide où elle est mesurée.

\subsubsection{Origine du $\mathrm{CO}_{2}$}

Le dioxyde de carbone acquis par les eaux minérales dans leur circulation souterraine peut avoir trois origines :

- une origine magmatique (dite aussi endogène, profonde, «juvénile » ou mantellique) avec un $\mathrm{CO}_{2}$ dont les caractéristiques isotopiques (enrichissement en 13C), le cortège d'autres gaz qui l'accompagnent $(\mathrm{He}, \mathrm{H}, \ldots)$ le mode d'émanation et parfois la température sont semblables aux gaz volcaniques. Cela ne signifie pas que les émissions de $\mathrm{CO}_{2}$ mantellique soient limitées aux zones de

\section{Exploitation of carbonated mineral waters}

The particular chemistry of these waters, which are naturally highly unstable, means that special exploitation processes are necessary. Detailed study of their thermodynamic and geochemical behaviour involving measurement, analysis, additional research and modelling provides the essential data for designing the systems needed for production, transport, treatment, storage and bottling.

Following some general remarks on these resources, the theoretical and practical implications of these natural constraints are described, indicating the 2-phase aspects of the flow patterns and the necessary control of the resources, the gas and the appropriate treatments.

The case of Montrond-les-Bains is described at the end of the paper. 
volcanisme actif: les fractures profondes, les zones à magmatisme récent par exemple peuvent produire ce type de gaz,

- une origine sédimentaire (thermodécarbonatation par métamorphisme) dans des régions où le flux de chaleur profond est suffisamment élevé pour transformer les niveaux carbonatés enfouis en d'autres minéraux (zéolites, épidote, plagioclases,...),

- une origine biogénique (exogène, atmosphérique) résultant de l'évolution de matière organique (photosynthèse, oxydation d'hydrocarbures,...). Le $\mathrm{CO}_{2}$ est alors accompagné d'autres gaz souvent plus abondants (azote, argon,...) et les signatures isotopiques sont caractéristiques.

Les eaux minérales carbo-gazeuses du territoire français ne se répartissent pas également suivant ces trois origines.

\subsubsection{Les provinces carbo-gazeuses de la France continen- tale}

\section{a) Le centre, le sud et l'est du Massif Central}

Cette zone renferme à elle seule la grande majorité des sources carbogazeuses françaises. Celles-ci se situent principalement en Auvergne (Limagne, Mont-Dore, Cézallier....). Forez, Bas-Vivarais et Languedoc (Escandorgue). Ces sources présentent toutes un $\mathrm{CO}_{2}$ d'origine profonde, lié à un volcanisme néogène et à un amincissement de la croûte terrestre.

\section{b) Le nord-est du Massif Central}

Entre la terminaison septentrionale du Sillon Houiller, le Morvan et même les Vosges une série d'eaux minérales renferment des gaz (dont du $\mathrm{CO}_{2}$ ) d'origine, au moins partiellement, exogène (BAUBRON et al., 1981).

c) Les Alpes

On regroupe dans cette province des sources de Tarentaise, du Briançonnais, du Dévoluy et de l’ouest de la Drôme pour la plupart situées sur des accidents majeurs.

\section{d) Autres sources carbogazeuses}

Dans le sud-ouest du Massif Central, l'ouest et l'est des Pyrénées et les bordures de la plaine d'Alsace existent, ponctuellement, des émergences carbogazeuses.

\subsubsection{Gamme de composition}

Les eaux minérales françaises présentent une grande diversité de composition puisque la concentration en bicarbonate, paramètre caractéristique de ces eaux qui peut servir à leur classement, se trouve compris entre moins de $200 \mathrm{mg} / \mathrm{l}$ (ex. : Ogeu-les-Bains) et plus de $4000 \mathrm{mg} / \mathrm{l}$ (ex. : Vichy). Contrairement à une idée reçue, le taux de gaz $n$ 'est pas techniquement un bon critère de classement. En effet l'impératif d'exploitation d'une eau minérale est de correspondre à une composition reconnue par le Ministère de la Santé, composition généralement fondée sur la qualité de cette eau dans les exutoires historiques. Or si ceux-ci ont montré les qualités intrinsèques de ces eaux, ils n'ont pas obligatoirement respecté le rapport naturel des flux d'eau et de gaz souterrains.

La mise en solution du $\mathrm{CO}_{2}$, à une certaine étape de la circulation souterraine de l'eau minérale modifie profondément ses propriétés chimiques ; cela se traduit dans un premier temps par une charge accrue en bicarbonates et par une acidification rendant le fluide plus agressif vis-à-vis de son environnement minéral : le fluide va alors se charger en divers éléments $(\mathrm{Ca}, \mathrm{Fe}, \mathrm{Mn}, \mathrm{Al}, \ldots$ ) aboutissant à des minéralisations globales plus élevées pour les eaux minérales carbogazeuses (l'éventail des résidus secs va de 180 à $6375 \mathrm{mg} / \mathrm{l}$ ) que pour les eaux plates (résidus secs compris entre 37 et $3509 \mathrm{mg} / \mathrm{l}$ ).

\subsection{L'auto gas-lift}

\subsubsection{Dégazage et évolution physico-chimique (LEITAO ROBALO et al., 1990)}

Si l'on part d'une ressource en eau de faciès carbogazeux, monophasique au niveau de l'aquifère, au cours de la remontée vers la surface la pression va baisser ; ce fluide passera alors généralement par une zone où le gaz s'exprimera : il s'agit du franchissement de la courbe appelée point de bulle en thermodynamique. A partir de cet instant l'écoulement dans la complétion du forage devient diphasique. Cette évolution amène deux phénomènes principaux: le premier est une chute progressive de masse volumique en remontant vers la surface, le second est une évolution physico-chimique des fluides en présence.

Ces deux phénomènes peuvent avoir lieu naturellement dans les sources préexistantes aux forages : cependant les pertes de charges généralement beaucoup plus importantes en contexte naturel limitent le dégazage souterrain et permettent l'expression majoritaire du gaz dans des zones proches de la surface où peuvent se déposer alors des travertins.

Tout ceci montre le risque de laisser un forage d'eau carbogazeuse en production sans connaissance précise de son hydrodynamique et de sa thermochimie. Des colmatages rapides de colonne de production, de pompes et de toute l'exploitation en aval peuvent ainsi se produire.

\subsubsection{Répercussion sur l'activation de puits (HONEGGER, 1985)}

D'un point de vue technique ce dégazage induit un auto gas-lift, en comparaison avec les procédés classiques de stimulation de puits très utilisés en milieu pétrolier et au cours des pompages d'essais de forage d'eau. En effet la réduction naturelle ou artificielle de masse volumique de l'eau par apparition et coalescence de bulles est un outil puissant de stimulation des puits à pression de réservoir constant.

\subsubsection{L'adaptation des mesures}

Le caractère diphasique des eaux induit de nombreuses erreurs ou artefacts dans les mesures.

Tout d'abord les informations de pression de réservoir, essentielles dans le cadre de toute exploitation d'eau souterraine doivent être pondérées par l'état thermodynamique des fluides au niveau de cette mesure. Ceci concerne particulièrement les mesures réalisées en fond de puits. Mais toutes les autres mesures (conductivité, débit,... au moyen d'électrodes de sécurité et même de vibration) doivent être corrigées en fonction de ce paramètre.

Par ailleurs, les prélèvements et analyses de fluide nécessitent aussi des procédures et matériels particuliers, que 
ce soit des séparateurs de phase, des mesures sous pression, et des analyses in situ pour les espèces instables (gaz dissous, bicarbonates).

\subsection{Des techniques de prospection spécifiques}

La présence de gaz carbonique en excès dans les eaux profondes amène généralement des circulations de gaz, après séparation partielle dans les couches superficielles du sol. Ceci peut se révéler un indicateur précieux des circulations de l'eau recherchée. Des techniques de prélèvements et d'analyse du $\mathrm{CO}_{2}$ dans les sols ont été développées pour réaliser des campagnes légères de prospection et fournir des cartographies précises et rapides de ces circulations. Ces résultats ne sont certainement pas suffisants pour implanter des ouvrages de production, mais permettent des recoupements avec d'autres informations, en particulier une autre prospection avec mesure des teneurs en Radon dans les sols ; ce gaz trace souvent des remontées d'eaux profondes et présente des caractéristiques de percolation différentes, permettant un recoupement d'informations ainsi que des réponses complémentaires.

\subsection{Des systèmes d'exploitation adaptés}

\subsubsection{Activation de puits}

Le gas-lift est un excellent procédé de stimulation de puits, très largement employé en milieu pétrolier (il s'agit alors de gas-lift induit, cf. fig. 1). Dans le métier de production d'eau, son utilisation présente deux inconvénients principaux :

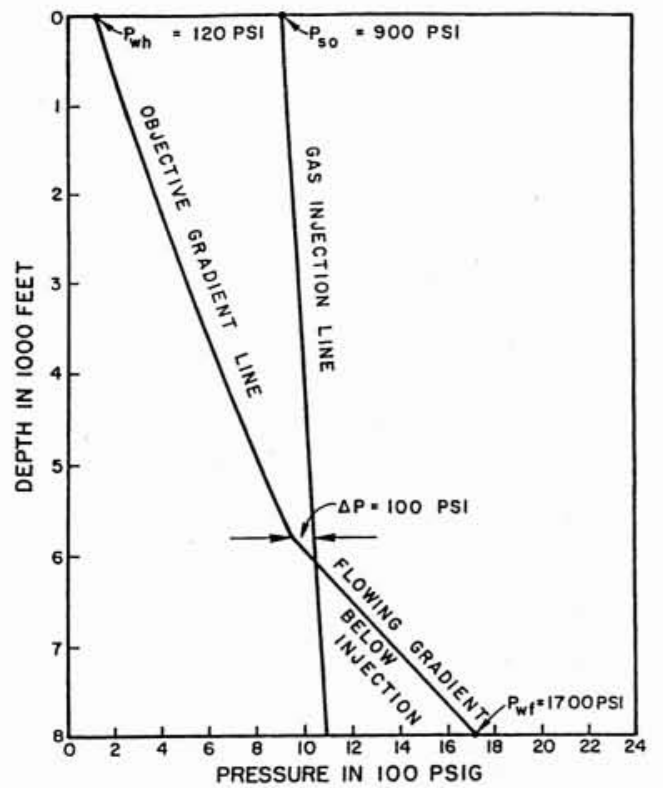

1. Construction d'une courbe de gas-lift.
- un coût relativement élevé en raison du matériel de compression et de séparation de gaz en comparaison avec des pompes centrifuges immergées ;

- un rendement énergétique assez faible et difficile à optimiser surtout dans des phases transitoires de production.

La grande difficulté de dimensionner les conditions optimales de production par gas-lift (naturel ou induit) est aussi à l'origine de son utilisation très discrète. Un des problèmes principaux réside dans le maintien de la production selon un mode d'écoulement constant, et ce malgré les modifications systématiques des caractéristiques d'un réservoir en production. Il en résulte parfois après une période d'écoulement homogène, des phénomènes de type geyser (production intermittente correspondant à un écoulement dit «à bouchons »), qui ne sont plus compatibles avec les besoins.

Aussi a-t-on généralement affaire soit à des puits suffisamment artésiens jaillissants pour être exploités en auto gas-lift, cas relativement exceptionnel, soit à l'utilisation de pompes immergées. Dans ce cas le dimensionnement des caractéristiques de ces pompes, mais aussi leur positionnement dans le forage vis-à-vis des conditions thermodynamiques du fluide doivent être très soigneusement calculés.

\subsubsection{Gestion des annulaires de puits}

L'annulaire de production, entre casing de puits et colonne de production est aussi, dans ce cas un lieu très particulier : soit il est maintenu hermétique avec une pression de gaz conséquente (à contrôler pour éviter un dénoyage de pompe immergée), soit il est en décharge tarée vers l'extérieur.

\subsubsection{Transport et stockage des eaux}

En ce qui concerne le transport de ces eaux, plusieurs configurations sont à envisager, toujours fonction des conditions thermochimiques en tête de puits, mais aussi des besoins exprimés par l'exploitant en terme de quantité de gaz et de risque d'entartrage :

- soit une séparation partielle ou totale du gaz est provoquée à proximité de l'ouvrage de production, le transport de l'eau devenant une simple adduction;

- soit le transport concerne l'eau et le gaz et il s'agit de maintenir un écoulement monophasique pour éviter des poches de gaz, des pertes de charges conséquentes et des coups de bélier.

\subsubsection{Traitement des fluides}

Les eaux carbogazeuses, du fait de leur faible $\mathrm{pH}$, ont une tendance à se charger en ions métalliques, particulièrement en fer et manganèse. Au cours de leurs captages, le moindre contact avec des eaux plus superficielles, plus oxygénées où même le simple contact atmosphérique provoque à plus ou moins long terme une oxydation et des boues d'hydroxydes. Ces phénomènes doivent être contrôlés dès la conception du forage par la connaissance précise des horizons captés, mais aussi par des équipements d'exploitation particuliers jusqu'à une unité de traitement. Cette 
dernière doit être réalisée dans le cadre strict des procédés de traitement admis pour les eaux minérales.

\subsubsection{Analyse des risques}

La présence de dioxyde de carbone dans l'eau (ou après séparation) amène une réflexion quant :

- aux risques intrinsèques venant de ce gaz dans les locaux d'exploitation, les têtes de puits et principalement dans toutes les zones en points bas non aérées :

- à la sélection des matériaux au contact soit de l'eau chargée de $\mathrm{CO}_{2}$, soit du gaz lui-même particulièrement avec de l'humidité.

\subsubsection{Cas d’un réservoir diphasique}

Quelques ressources d'eaux carbogazeuses sont déjà diphasiques au niveau de la ressource captée, aussi y a-t-il lieu dans ces cas d'établir un parallèle avec les prospections de pétrole où cette configuration est classique, et prévoir :

- des organes de prévention d'éruption (bien que ceci devrait toujours être le cas) ;

- la poursuite du forage jusqu'à la base des horizons producteurs d'eau ;

- la fermeture de tout exutoire de production de gaz, dont la poche représente la pression potentielle de la ressource en eau.

\section{II —UN EXEMPLE CARACTÉRISTIQUE}

\subsection{Eau minérale «Geyser-Détente " à Montrond-les- Bains}

Le site de Montrond-les-Bains occupe une position centrale dans le fossé d'effondrement du Forez. Celui-ci s'est formé à l'oligocène dans le cadre de la tectonique distensive et du volcanisme qui lui est associé sur le pourtour de l'arc alpin.

Dans le Massif Central, ces grabens expriment une remontée du Manteau. Le volcanisme ponctuel et peu différencié du Forez se répartit essentiellement sur la bordure ouest du Bassin comme certaines autres sources d'eaux minérales. Le remplissage sédimentaire du fossé (alternance de séries argileuses et sablo-gréseuses) peut atteindre $700 \mathrm{~m}$; à Montrond il dépasse les $500 \mathrm{~m}$ du forage «Geyser» qui aboutit dans des passées argileuses. Les eaux remontent à travers le socle granitique par la fracturation liée aux failles normales du fossé.

\subsection{Une physico-chimie exceptionnelle}

Les eaux minérales du Forez appartiennent soit à une famille alcaline soit à une famille carbogazeuse; c'est clairement dans le second groupe que se rangent les eaux de Montrond (Foulllac et al., 1982). Celles-ci se distinguent (tabl. l) par un caractère exceptionnellement bicarbonaté et carbogazeux : $\mathrm{HCO}_{3}$ représente $71 \%$ de la composition chimique de l'eau et, à $35^{\circ} \mathrm{C}$, il y a $20,5 \mathrm{~g}$ de $\mathrm{CO}_{2}$ libre/l auxquels il faut ajouter $0,5 \mathrm{~g} / \mathrm{l}$ de $\mathrm{CO}_{2}$ encore dissous. $\mathrm{Ce} \mathrm{CO}_{2}$ représente $99,4 \%$ de la phase gazeuse (LeITAO-Robalo, 1990).

L'origine profonde du fluide est en outre attestée par : - les dosages isotopiques du carbone $(13 \mathrm{C})$, du soufre ( $34 \mathrm{~S})$, du tritium $(3 \mathrm{H})$,

- les fortes teneurs en bore, ammonium $\left(\mathrm{NH}_{4}^{+}\right)$et arsenic.

D'autres particularités géochimiques existent (FOUILLAC 1982) : pauvreté en $\mathrm{Cl}$, en alcalins lourds ( $\mathrm{K}, \mathrm{Rb}, \mathrm{Cs}$ ), en $18 \mathrm{O}$ (échange entre l'oxygène de l'eau et celui du $\mathrm{CO}_{2}$ ).

\subsection{Simulation du comportement thermochimique}

Les géothermomètres calculés sur la base des concentrations en alcalins doivent tenir compte des éventuelles réactions d'échange entre le fluide et des minéraux argileux lors de la remontée moyennant quoi la température profonde d'équilibre est estimée à $150^{\circ} \mathrm{C}(\mathrm{Na} / \mathrm{K}$ corrigé $)$, ce que confirme le géothermomètre à quartz conductif (KHARAKA et al., 1988).

En ce qui concerne le point de bulle, une mesure directe (par densité optique) donne une valeur de 11,5 bars relatifs sur le fluide du forage ; sur ce même fluide, le calcul par deux logiciels distincts produit des résultats voisins :

- TP-DEGAZ (CZERNICHOWSKI-LAURIOL, 1988) donne 10,7 bars

- Solmineq 88 (KhaRAKA et al., 1988) 12,4 bars.

Si maintenant on calcule les équilibres minéraux/fluide par exemple au moyen du logiciel Solmineq 88 (KHARAKA et al., 1988), on constate que (tabl. 2):

- le fluide dégazé est sursaturé en oxy-hydroxydes de fer, - la remise en solution de l'ensemble du $\mathrm{CO}_{2}$ libéré en surface ne suffit pas à ramener le fluide à l'équilibre avec les carbonates (calcite, dolomite et sidérite sont toujours sursaturées).

On peut alors calculer la quantité de $\mathrm{CO}_{2}$ nécessaire pour parvenir à cet équilibre ; la même modélisation indique alors que 1,28 mole de $\mathrm{CO}_{2}$ doit être ajouté par litre d'eau, soit une pression partielle de 51,6 bars de $\mathrm{CO}_{2}$

Tableau 1. - Analyse chimique des éléments majeurs de l'eau minérale de Montrond-les-Bains.

\begin{tabular}{|c|c|c|c|c|c|}
\hline $\mathrm{HCO} 3$ & $\mathrm{Cl}-$ & SO4- - & $\mathrm{Ca}++$ & $\mathrm{Na}+$ & $\mathrm{K}+$ \\
\hline 12200 & 136 & $<1$ & 119 & 4440 & 112 \\
\hline
\end{tabular}

\begin{tabular}{|c|c|c|c|c|}
\hline$\sqrt{\mathrm{Mg}++}$ & $\mathrm{Fe}++$ & $\mathrm{Mn++}$ & $\mathrm{SiO} 2$ & Minéral.tot. \\
\hline 43 & 1 & 0.04 & 87 & 17146 \\
\hline
\end{tabular}


Tableau 2. - Etat de saturation du fluide de Montrond-les-Bains à diverses pressions de $\mathrm{CO}_{2}$.

\begin{tabular}{|c|c|c|c|}
\hline Minéraux & $\begin{array}{r}\text { Fluide de surface dégazé } \\
\log (\text { P.A.I./Ks })\end{array}$ & $\begin{array}{r}\begin{array}{r}\text { Fluide de surface regazé } \\
\log (\text { P.A.I./Ks })\end{array} \\
\end{array}$ & \begin{tabular}{|l|l|}
$\begin{array}{ll}\text { Fluide à l'équilibre avec la } \\
\text { dolomite } \quad \log (\text { P.A.I./Ks })\end{array}$ \\
\end{tabular} \\
\hline Calcite & 1.58 & 0.15 & $<$ \\
\hline Dolomite & 4.69 & 1.82 & 0.06 \\
\hline Magnésite & 0.97 & $<$ & $<$ \\
\hline Sidérite & 1.87 & 0.42 & $<$ \\
\hline & & & \\
\hline Chalcédoine & 1.16 & 1.16 & 1.18 \\
\hline Quartz & 1.48 & 1.49 & 1.51 \\
\hline Goethite & 0.72 & $<$ & $<$ \\
\hline Magnétite & 0.82 & $<$ & $<$ \\
\hline
\end{tabular}

P.A.I. : produit d'activité ionique.

Ks : produit de solubilité.

(toujours à la température d'émergence de $37,5^{\circ} \mathrm{C}$ ). Comme le point de bulle réel ne peut être à une pression inférieure à celle de l'équilibre calco-carbonique, on en déduit :

- que les points de bulle mesurés et calculés sur le fluide du forage ne sont qu'apparents,

- que la pression partielle de $\mathrm{CO}_{2}$ dans le réservoir est supérieure à 52 bars,

- qu'une partie non négligeable du $\mathrm{CO}_{2}$ a dégazé en profondeur sans être capté à la production (ce que semble confirmer l'important dégazage constaté dans l'annulaire du forage),

- que le fluide est déjà d'une part diphasique, d'autre part sursaturé au niveau du pompage et que seule la cinétique explique l'absence de dépôts à ce niveau.

L'exemple de Montrond-les-Bains, s'il est exceptionnel par ses teneurs en bicarbonate et en $\mathrm{CO}_{2}$, montre un phénomène classique de dégazage avec ses conséquences. L'importance de l'approche géochimique est ici soulignée tant au niveau de la qualité des mesures et des prélèvements de terrain que de la modélisation ultérieure.

\section{III $\square$ CONCLUSION}

L'exploitation des eaux minérales carbogazeuses demande toute l'attention nécessaire à des eaux de grande qualité, mais nécessite en plus de nombreuses spécificités de conception.

C'est essentiellement la connaissance précise du comportement hydrochimique de ces eaux depuis leur ressource souterraine jusque dans l'exploitation qui permet de spécifier tous les équipements à mettre en place, ainsi que les procédures de fonctionnement. Trop d'interventions sont encore constatées a posteriori induisant des pertes d'exploitations et des réinvestissements dans ce domaine aujourd'hui techniquement maîtrisé.
Le secteur des eaux carbogazeuses embouteillées est en plein développement depuis trois ans avec l'apparition sur le marché des eaux "La Salvetat ", "Arvie », ainsi que le fort développement de « Vernet », « Ste Marguerite », « La Vernière ", « Parot ", « Arcens ».

\section{Bibliographie}

Baubron J.C., Batard F., Bosch B., Marce A. \& Risler J.J. (1981). - Isotopic identification of gases from deep origin in french thermomineral waters, soumis à $\propto$ Journal of volcanology and geothermal research ".

CZERNICHOWSKI-LAURIOL I. (1988). - Modélisation de l'évolution de la chimie des fluides géothermaux lors de leur exploitation par forages. Thèse doctorat de I'Institut National Polytechnique de Lorraine, Document du BRGM $\mathrm{n}^{\circ} 159,196 \mathrm{p}$.

Fouillac C., Bosch B., Degranges P., Fouillac A.M., MERCIER-BATARD F. \& SARCiA C. (1982). - Etude géochimique des sources minérales de la Limagne de Loire. conséquence en prospection géothermique, Rapport BRGM à la C.C.E.

HONEGGER J.L. (1985). - Etude d'un échangeur à contact direct co-courant pour la valorisation thermodynamique de la géothermie moyenne enthalpie, Document du BRGM $\mathrm{n}^{\circ} 98$.

Kharaka Y.K., GunTer W.D., AgGarwal P.K., PERKINS E.H. \& DEBRAAL J.D. (1988). - Solmineq 88: A computer program for Geochemical Modeling of Water-Rock Interactions, U.S. Geological Survey Water Resources Investigations Report 88-4227, 420 p.

Lettao Robalo J.M., Honegger J.L. (1990). - Evolution des caractéristiques physico-chimiques des eaux carbogazeuses depuis le sous-sol jusqu'à l'exploitation, Hydrogéologie $n^{\circ} 4$.

RAMBAUD A. (1992), - Les eaux conditionnées, In : Les eaux conditionnées de D. Tampo, Tec \& Doc Lavoisier, Paris, $175 \mathrm{p}$. 